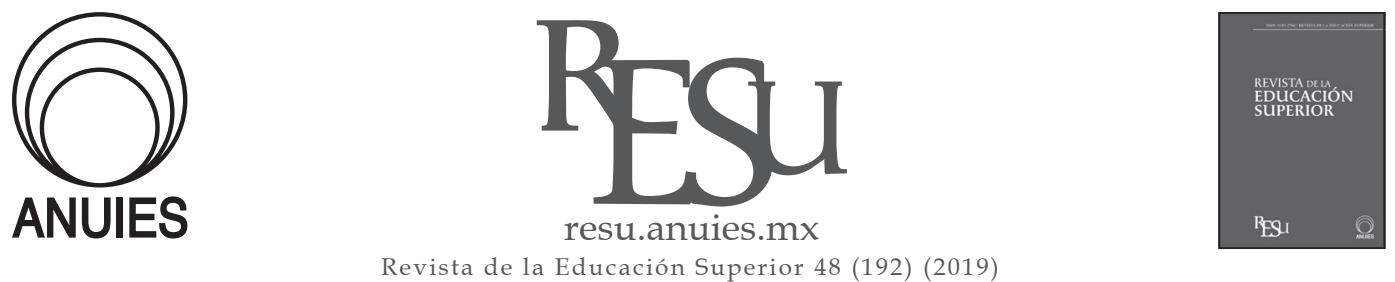

ARTÍCULO

\title{
Estrategias de permanencia y desigualdad social en estudiantes universitarios de Córdoba-Argentina en la actualidad
}

\author{
Strategies of permanence and social inequality in university students of \\ Córdoba-Argentina at present
}

\author{
Manuel Alejandro Giovine y Ana Antolin Solache* \\ *Universidad Nacional de Córdoba, Argentina, \\ Correo electrónico: giovine.manuel@gmail.com
}

Recibido el 21 de noviembre del 2018; aceptado el 10 de octubre del 2019

\begin{abstract}
Resumen
A cien años de la Reforma Universitaria de Córdoba-Argentina, ponemos en discusión algunos desafíos frente a la democratización universitaria. Nos proponemos dar cuenta de las estrategias que establecen los alumnos universitarios ante la dificultad de realizar sus estudios en el tiempo previsto. Estas estrategias se diferencian en función del volumen y la estructura de los capitales puestos en juego por sus familias. Para ello, presentamos los instrumentos de reproducción disponibles, nos valemos del enclasamiento de las familias de Córdoba para caracterizar su acceso diferencial por clase a la universidad y describimos los sentidos vividos asociados a las estrategias de permanencia universitaria por clase: estrategias de persistencia y de sustitución.
\end{abstract}

Palabras clave: Desigualdad educativa; Estrategias de permanencia; Educación universitaria; Clase social; CórdobaArgentina

0185-2760/@ 2016 Asociación Nacional de Universidades e Instituciones de Educación Superior A.C. Este es un artículo Open Access bajo la licencia CC BY-NC-ND (http://creativecommons.org/licenses/by-nc-nd/4.0/). 


\begin{abstract}
One hundred years after the University Reform of Córdoba-Argentina, we are discussing some challenges facing university democratization. We intend to give an account of the strategies established by university students viewing the difficulty in completing their studies in the established time. These strategies differ according to the volume and structure of the investment done by their families. To do this, we present the available reproduction instruments, we use the classification of the Córdoba families to characterize their differential access to the university by social class and describe the lived senses associated with the university permanence by social class: persistence and substitution strategies.
\end{abstract}

Keywords: Educational inequality; Permanency strategies; University education; Social class; Córdoba-Argentina

\title{
Introducción
}

A 100 años de la Reforma Universitaria, la actualidad de los principios que la guiaron se reaviva. Este momento histórico ha sido considerado como precursor en la democratización de la educación argentina, entre otras razones por la apertura de los claustros a la clase media (Chiroleu, 2009; Suasnábar, 2009). No es nuestro objetivo detenernos al inicio del siglo xx sino más bien entrar en el debate de un conjunto de políticas "democratizadoras" llevadas a cabo en las últimas décadas y sus efectos a 100 años de la reforma de 1918.

En el comienzo del siglo XXI continúa la expansión del sistema educativo argentino, dado principalmente por la ampliación de la cobertura en todos los niveles (García de Fanelli, 2015; Miranda, 2013; Rivas et al., 2010; Acosta, 2009). Esta ampliación se da en consonancia con la implementación de la obligatoriedad de los niveles inicial y secundario (Dirección Nacional de Información y Estadística Educativa, Argentina, 2016; Nobile, 2016). El nivel superior no ha sido ajeno a este proceso y también ha crecido de manera sostenida, incluyendo a familias de clase media (García de Fanelli, 2015; Brunner, 2012) en particular mujeres que venían incrementando su participación desde mediados del Siglo xx (Brunner, 2012; Palermo, 2006).

Investigaciones recientes muestran un incremento en la oferta de nivel universitario a lo largo del siglo xx y principios del xxI, con periodos de mayor o menor intensidad en función de los contextos políticos y sociales atravesados en el país (García de Fanelli, 2015, 2005; Brunner, 2012). Este proceso de expansión de la oferta y de la demanda de nivel superior se manifiesta de un modo diferencial en las estrategias de las familias de Córdoba. 
A pesar del aumento sostenido en la matrícula a nivel nacional y local (Ministerio de Educación de la Nación, 1996-2014), tanto en el sector privado como en el estatal (Gutiérrez y Giovine, 2017; García de Fanelli, 2015), el acceso al nivel universitario para las familias de clase baja y media empobrecida sigue siendo muy restrictivo (Gutiérrez y Giovine, 2017), fundamentalmente porque no logran terminar el secundario (Jiménez Zunino y Giovine, 2017).

Las dificultades para los "recién llegados", las primeras generaciones de estudiantes universitarios, no surgen tanto al momento del ingreso como durante el cursado. Esto se evidencia en los indicadores de desgranamiento y deserción cada vez más altos, particularmente en el sector estatal (García de Fanelli, 2015).

Los ingresantes y los egresados aumentan en menor proporción que los alumnos universitarios, lo que pone en relieve el tema que abordamos en este artículo: ¿Quiénes llegan a la universidad en Córdoba? ¿Quiénes son los que se retrasan o abandonan? ¿Los que abandonan una carrera, continúan sus estudios? ¿Qué estrategias articulan, los alumnos y sus familias, para sostenerse en el nivel? ¿Son las mismas estrategias para las diferentes clases sociales?

Con un conjunto de datos disponibles del Censo Nacional de Población, Hogares y Viviendas (CNPHyv) y de la Encuesta Permanente de Hogares (EPH), más las entrevistas realizadas en el marco del equipo de investigación del que formamos parte y de entrevistas de nuestras investigaciones de maestría y doctorado, exploramos y describimos el fenómeno de la dilación de los estudios universitarios en Córdoba-Argentina para el periodo 2003-2015. ${ }^{1}$

\section{El nivel universitario en Argentina}

Al igual que en toda Latinoamérica, la matrícula de la educación superior en Argentina se ha expandido desde principios del siglo xx y sostiene su crecimiento en lo que va del siglo XxI. Diversos autores muestran este crecimiento (García de Fanelli, 2015, 2005; Brunner, 2012; Gorostiaga, 2012) que García de Fanelli precisa para Argentina hasta inicios de este siglo en una tasa de crecimiento promedio de un 7\% anual (García de Fanelli, 2005).

Uno de los efectos de este crecimiento es una tasa bruta de escolarización superior de un 52,6\% en 2011, para los jóvenes entre 18 y 24 años. Si tomamos

\footnotetext{
${ }^{1}$ Retomamos el concepto de dilación en el estudio presentado por Stasiejko et al. (2013). Utilizamos el concepto de dilación en este estudio para referirnos específicamente al fenómeno en el cual el estudiante, matriculado en un determinado plan de estudios, no aprueba la cantidad de materias previstas por año, o en el tiempo teórico de la carrera, retrasando el egreso.
} 
sólo los estudiantes universitarios, la tasa bruta de educación universitaria entre 18 y 24 años es de 37,8\% (Ministerio de Educación de la Nación, 2011).

Este proceso fue acompañado por el aumento de instituciones educativas de nivel universitario de manera sostenida desde la vuelta a la democracia en Argentina, con similar intensidad entre las universidades estatales y privadas (Gutiérrez y Giovine, 2017; García de Fanelli, 2015). ${ }^{2}$ No obstante esto, las universidades estatales siguen siendo más voluminosas que las privadas, con 4,21 veces más alumnos promedio por institución en 2011 (Secretaría de Políticas Universitarias-SPU).

La condición de posibilidad de las elevadas tasas brutas se debe, en parte, a un aumento sostenido del egreso del nivel secundario (Gorostiaga, 2012; García de Fanelli, 2005) y en parte a una mayor permanencia de los estudiantes en el nivel. No obstante ello, las familias de clase media y baja siguen teniendo dificultades para que sus miembros accedan al nivel universitario, dado que todavía les resulta difícil finalizar la educación secundaria (Jiménez Zunino y Giovine, 2017; Gorostiaga, 2012; Rivas et al., 2010; Jacinto, 2006).

Para quienes no finalizaron sus estudios secundario las universidades estatales han generado un sistema de ingreso por el cual quienes tengan más de 25 años y aprueben un examen de competencias básicas de matemáticas y lectoescritura pueden estudiar en la universidad. ${ }^{3}$

Tomando datos del cnPHyv 2010, sabemos que un 14,5\% de la población de 25 años y más cursa o cursó el nivel universitario. Estos datos ponen de relieve cuán limitado sigue siendo para el total de la población el acceso a la universidad, especialmente a la universidad estatal. Además, los resultados del censo son contundentes respecto de los egresados, sólo un 8,3\% de quienes tienen 25 años y más han finalizado los estudios universitarios.

Para apreciar mejor estos procesos en el período de interés, hemos buscado un proxi comparando los egresados de un año con los ingresantes de cinco años anteriores y, de un modo similar a García de Fanelli (2015) y Gutiérrez y Giovine (2017), calculamos una aproximación a la tasa de egreso específica de la población universitaria. Tomando como año de referencia para los ingresantes 2011 y como año de referencia para los egresados 2015, se observa una proporción de egresados de un 30\% para todo el nivel universitario argentino. Si tomamos las universidades estatales ese porcentaje disminuye al

\footnotetext{
${ }^{2}$ En este artículo al mencionar universidades nos referimos tanto a universidades como a institutos universitarios.

${ }^{3}$ Esta disposición es producto de la Ley de Educación Superior 24521/1995 que prevé en su artículo $7^{\circ}$ la posibilidad de ingreso a la universidad (en carreras de pregrado y grado) de aspirantes que tengan el primario completo.
} 
$27 \%$ y las privadas alcanzan un $40 \%$ de egresados en proporción a los ingresantes (Ministerio de Educación de la Nación, 2011-2015). Es posible que la diferencia en el egreso del sector estatal frente al privado se deba, entre otros factores, al costo de la matrícula (García de Fanelli, 2015) y al mayor nivel de ingresos de los alumnos de las universidades privadas (Ennis y Porto, 2001).

Los datos de reinscriptos netos ${ }^{4}$, nuevos inscriptos y egresados en la educación universitaria estatal en Argentina ha crecido de manera sostenida desde la vuelta a la democracia a fines de 1983, con la apertura del acceso a la universidad (ver Gráfico 1). Se observa un crecimiento mayor para los reinscriptos netos (que crecen 2,8 veces entre 1984 y 2015) que para los nuevos inscriptos (que aumentan un 1,6 veces de 1984 a 2015) y los egresados (que aumentan un 1,9 veces entre los años mencionados). El aumento sostenido y con mayor pendiente de los reinscriptos netos estaría mostrando la acumulación de quienes transitan sus estudios en un tiempo teórico de cursado mayor al previsto, dando cuenta del esfuerzo por sostener la permanencia en el nivel universitario.

Gráfico 1: Reinscriptos netos, nuevos inscriptos y egresados para las universidades estatales, total país, 1984-2015.

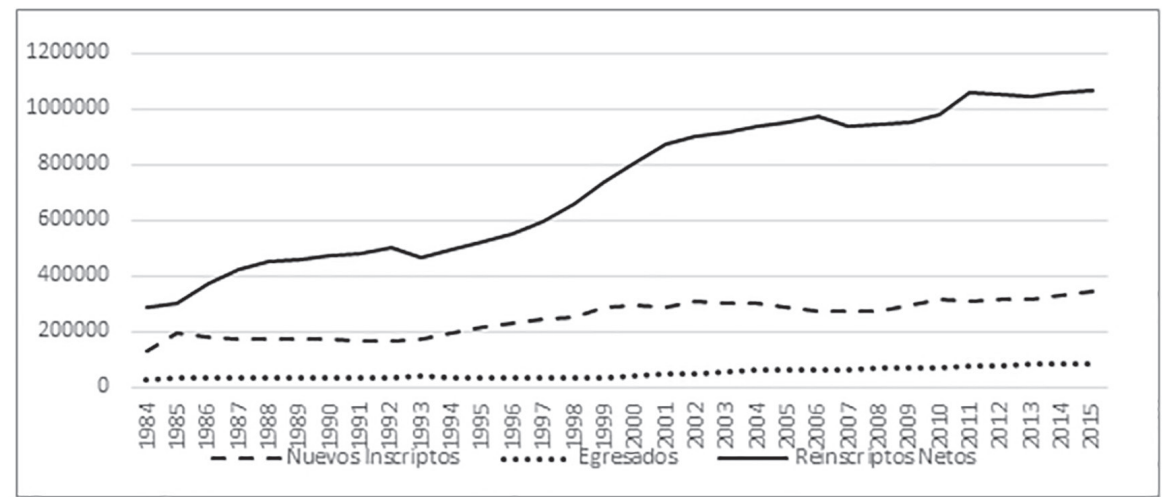

Fuente: elaboración propia en base a datos de Secretaría de Políticas Universitarias (SPU).

Para los reinscriptos netos, nuevos inscriptos y egresados en educación privada contamos con datos desde 1995 (ver Gráfico 2) donde también se observa un crecimiento sostenido. En este caso los reinscriptos netos y los nuevos ingresantes aumentan 1,9 veces entre 1995 y 2015, mientras que los egresados lo hacen en una proporción próxima a 4 (3,97 veces) en el periodo

\footnotetext{
${ }^{4}$ De aquí en adelante, denominamos reinscriptos netos a la variable Reinscriptos menos los Egresados de un año del Sistema de Información Universitario nacional.
} 
considerado. Nuestra hipótesis es que el mayor incremento en los egresados de las universidades privadas combina una mayor tasa de egresados de estas instituciones con la creación de muchas de ellas en la década de 19905, que tendrán sus graduados comenzado el nuevo siglo.

Gráfico 2: Reinscriptos netos, nuevos inscriptos y egresados para las universidades privadas, total país, 1995-2015.

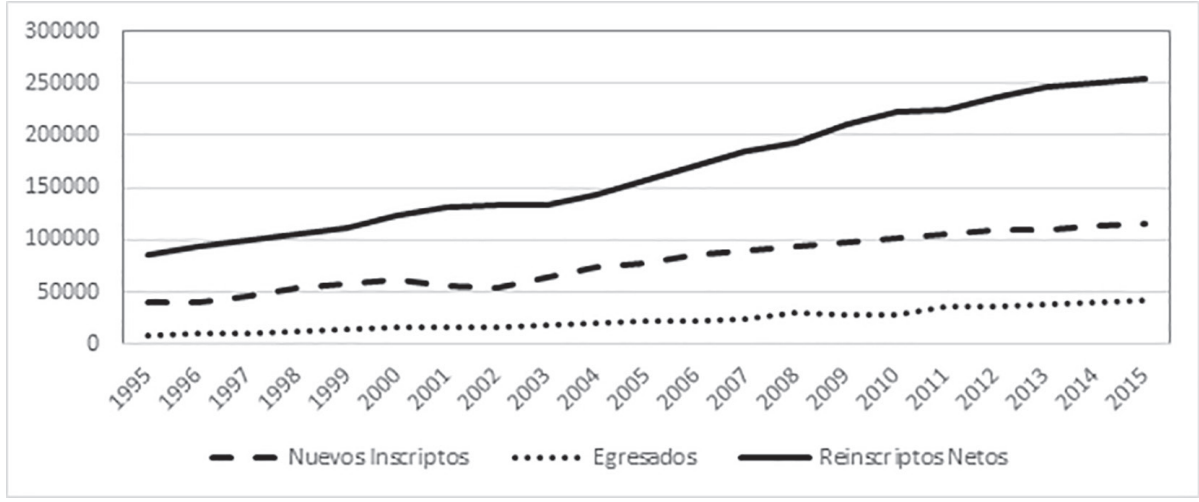

Fuente: elaboración propia en base a datos de Secretaría de Políticas Universitarias (SPU).

Si bien en ambos sectores se observa un crecimiento entre 1995 y 2015, es notoria la diferencia a favor de los reinscriptos netos, nuevos inscriptos y egresados de las universidades privadas (ver Tabla 1), lo que daría cuenta del proceso de expansión de la oferta educativa privada, a partir de la década de 1990, al igual que en toda América Latina (Brunner y Miranda, 2016).

Tabla 1: Incremento de reinscriptos netos, nuevos inscriptos y egresados de universidades estatales y privadas argentinas, 1995 y 2015 , en porcentaje.

\begin{tabular}{lll}
\hline $\mathbf{1 9 9 5}$ y $\mathbf{2 0 1 5}$ & Estatal & Privada \\
\hline Reinscriptos netos & 105,0 & 194,0 \\
\hline Nuevos inscriptos & 62,2 & 187,9 \\
\hline Egresados & 133,6 & 396,7 \\
\hline
\end{tabular}

Fuente: elaboración propia en base a datos de Secretaría de Políticas Universitarias (SPU).

Estaría contribuyendo a este proceso una oferta complementaria a la tradicional, diferenciada, con carreras alternativas y novedosas al principio del período. Además, el momento cualitativo del estudio nos permitió registrar

${ }^{5}$ Como muestra García de Fanelli (2015), las universidades e institutos privados en Argentina pasan de 23 en 1989 a 52 en 1999. 
la implementación de un modelo pedagógico que busca la retención y la graduación de los estudiantes a través de una amplia disponibilidad horaria y de una multiplicidad de modalidades semipresenciales y a distancia, que vienen a satisfacer una demanda que no lograban cubrir las universidades estatales.

En el siguiente apartado mostramos el devenir de estos procesos en el Gran Córdoba, a la vez que presentamos los instrumentos de reproducción específicos.

\section{La educación universitaria en Gran Córdoba}

La oferta educativa de nivel universitario en Gran Córdoba ${ }^{6}$ está compuesta por universidades dependientes del estado argentino, una universidad provincial creada recientemente y universidades privadas. Córdoba cuenta con la primera universidad estatal y la primera universidad privada autorizada a otorgar títulos habilitantes del país (Gutiérrez y Giovine, 2017; Giovine, 2015).

La oferta de universidades estatales está integrada por la Universidad Nacional de Córdoba (UNC) fundada por los jesuitas en 1613 y sede de la Reforma Universitaria de 1918), el Instituto Universitario Aeronáutico fundado en 1971, la Universidad Tecnológica Nacional-Facultad Regional Córdoba fundada en 1959 y la Universidad Provincial de Córdoba creada en 20077. Además, la Universidad Nacional de Villa María cuenta con una sede en la capital desde el año 2008.

Entre las universidades privadas señalamos en primer lugar a la Universidad Católica de Córdoba, fundada en 1956 y con licencia para otorgar títulos habilitantes desde 1959. A partir de la década de 1990 se crea un conjunto de universidades privadas no confesionales, la Universidad Blas Pascal, la Universidad Empresarial Siglo xxI y finalmente el Instituto Universitario de Ciencias Biomédicas de Córdoba que surge en 2012.

En Gran Córdoba también ofrecen sus certificaciones numerosas universidades estatales y privadas con sede en nuestro país y el extranjero, por lo general en convenio con instituciones locales (institutos de nivel superior,

\footnotetext{
${ }^{6}$ El aglomerado Gran Córdoba abarca a la ciudad de Córdoba y un conjunto de localidades del departamento Colón, al norte de la misma. Considerando el cNPHyv 2010 contaba con 1.454.536 habitantes y un área de 543,88 km2. Se corresponde con el área muestral de la EPH.

${ }^{7}$ Las carreras de la Universidad Provincial de Córdoba recibieron reconocimiento nacional a fines de 2017, por lo que sus datos no están incluidos en este estudio.
} 
fundaciones, organismos gubernamentales), mediante instancias formativas dictadas bajo las modalidades presenciales, semipresenciales y a distancia.

Datos de matrícula 2015 de la Secretaría de Políticas Universitarias, publicados por INDEC (2018), nos muestran que en la Provincia de Córdoba ${ }^{8}$ el $66,5 \%$ de los estudiantes universitarios está inscripto en universidades estatales y el 33,5\% restante en universidades privadas. Si consideramos a los nuevos inscriptos, el 46,6\% de ingresantes se inscribe en universidades privadas y sólo concentran el 28,7\% de los reinscriptos. El 40,2\% de los egresados de ese año lo ha hecho en estas instituciones privadas.

La población estudiantil de las universidades estatales y privadas presenta diferencias en su composición. La proporción de nuevos inscriptos en el total de estudiantes es más elevada en las universidades privadas que en las estatales: $37,4 \%$ en las primeras y $21,6 \%$ en las últimas. En relación con los reinscriptos, la proporción se invierte, mostrando que el 78,4\% de los estudiantes de las universidades estatales se concentra en esta condición, contra el 62,6\% en las privadas. El 6,5\% de los estudiantes de las universidades estatales egresan en el año 2015, mientras que en las universidades privadas esa proporción asciende al 8,7\%.

Tabla 2: Estudiantes, nuevos inscriptos, reinscriptos y egresados de universidades estatales y privadas, provincia de Córdoba, 2015.

\begin{tabular}{llll}
\hline Prov. de Córdoba & Estatales & Privadas & Total \\
\hline Estudiantes & 155.584 & 78.295 & 233.879 \\
\hline Nuevos Inscriptos & 33.633 & 29.308 & 62.941 \\
\hline Reinscriptos & 121.951 & 48.987 & 170.938 \\
\hline Egresados & 10.176 & 6.833 & 17.009 \\
\hline
\end{tabular}

Fuente: elaboración propia en base a Instituto Nacional de Estadísticas y Censos (2018), a partir de datos de la SPU.

Los datos del CNPHyv 2010 para la provincia de Córdoba nos permiten observar este mismo fenómeno desde la perspectiva de los sujetos. Del total de población mayor a 25 años (inclusive), sólo un 17,5\% accedió al nivel universitario y apenas un 10,0\% egresó del nivel ${ }^{9}$. Dentro de quienes tuvieron acceso

\footnotetext{
${ }^{8}$ Para la construcción de la tabla 2 y su lectura tomamos como unidad geográfica a la Provincia de Córdoba, dado que es la mejor aproximación disponible a los datos universitarios de Gran Córdoba. La población de Gran Córdoba representa un 41\% de la población de la provincia de Córdoba en el cnphyv 2010.

${ }^{9}$ Para esta sección incluimos en universitario completo a quienes informaron nivel de posgrado (completo e incompleto) y quienes informaron sólo universitario completo.
} 
al nivel, por grupos quinquenales de edad, se puede apreciar cómo aumenta la proporción de egresados conforme se incrementa la edad (ver Gráfico 3). Un 1,2\% de la población de 25 años y más tiene acceso al nivel de posgrado y apenas un $0,7 \%$ de la población lo ha finalizado en 2010.

También es posible distinguir dos grupos entre quienes no finalizaron sus estudios de grado: aquellos que abandonaron definitivamente y quienes no egresaron pero continúan asistiendo. La lectura de los datos advierte, en primer lugar, la disminución de la proporción de población que no finaliza el nivel, a medida que se incrementa la edad (ver Gráfico 3). En segundo lugar, resaltar la proporción de personas que declaran seguir asistiendo a la universidad en todos los grupos de edad, que disminuye rápidamente a partir del quinquenio 25-29 años, alcanzando un valor menor a $10 \%$ en el quinquenio 35-39. Esta población que declara seguir asistiendo conforma nuestro objeto de estudio.

Gráfico 3: Egresados, no egresados y no egresados que asisten al nivel universitario de la población mayor de 25 años por edad quinquenal, provincia de Córdoba, 2010. En porcentaje.

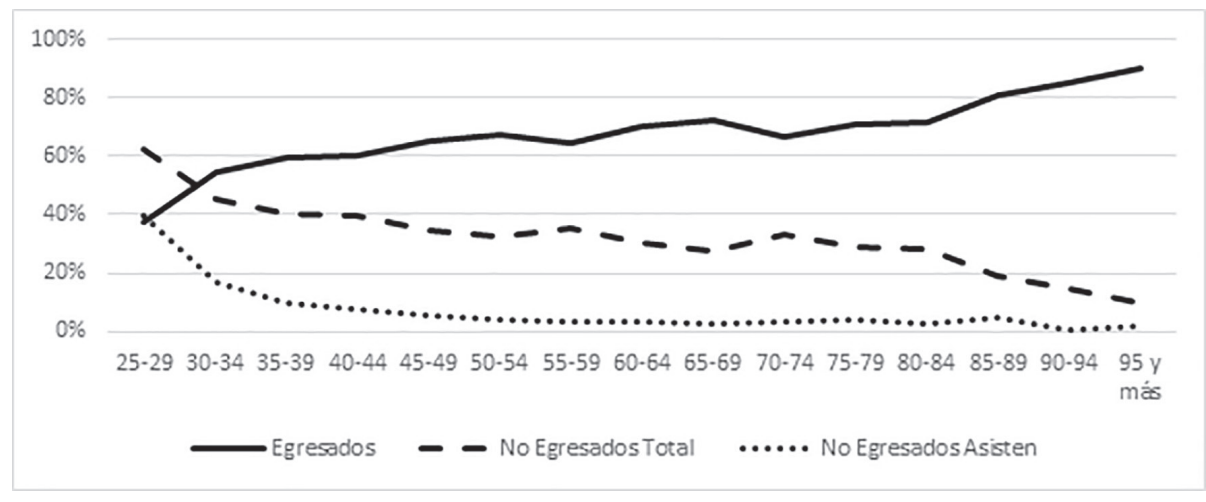

Fuente: elaboración propia en base a datos del CNPHyV 2010.

Comparando al interior de cada quinquenio vemos que, de 20 a 24 y de 25 a 29 años, se encuentra el mayor acceso a la universidad, cercano a un $27 \%$ de cada grupo de edad y alrededor de 70.000 casos por quinquenio. En los sucesivos lustros la proporción de personas con acceso a la universidad disminuye gradualmente.

Lo desarrollado hasta aquí nos permite apreciar la magnitud y la complejidad institucional de la educación universitaria en Gran Córdoba desde mediados del siglo xx y presentar los instrumentos de reproducción de nivel universitario disponibles. A continuación retomamos la construcción de clases y fracciones de clase (Gutiérrez y Mansilla, 2015) que agrupa a las fami- 
lias de Gran Córdoba en función del volumen y estructura de su capital y nos permite identificar y caracterizar a las familias con estudios universitarios.

\section{La desigualdad universitaria por clase en Gran Córdoba}

En este apartado describimos el acceso, la permanencia y el egreso diferenciales por clase social construida a la universidad en Gran Córdoba para los años 2003 y 2011. Nos valemos del Espacio Social de Gran Córdoba 2003 y $2011^{10}$, que toma como fuente de datos la Encuesta Permanente de Hogares (EPH) publicada por el Instituto Nacional de Estadísticas y Censos.

Nuestras unidades de análisis son las familias de Gran Córdoba. En la EPH la unidad de observación más próxima a la familia, como la entendemos en el equipo de investigación, son los hogares. Para la construcción del Espacio Social de Gran Córdoba se tomaron un conjunto de variables activas, atributos del referente de hogar $(\mathrm{RH})^{11}$, y el ingreso per capita familiar (IPCF) que permite distribuir el ingreso total familiar en la cantidad de miembros. También se ingresaron al análisis un conjunto de variables ilustrativas cuyas categorías aparecen representadas en el espacio social cuando están significativamente asociadas a las clases y fracciones de clase, pero no inciden en la deformación de dicho espacio (Gutiérrez y Mansilla, 2015).

A partir del análisis de correspondencias múltiples (ACM) y del método de clasificación jerárquica ascendente (CJA) se construyeron "clases en el papel” (Bourdieu, 1990) que aparecen en los gráficos 4 y 5. La selección de la cantidad de factores para la construcción de clases y la cantidad de clases y

${ }^{10}$ La construcción del Espacio Social se realizó en el marco del proyecto de investigación "Las clases y su reproducción en el espacio social cordobés (2003-2013)" dirigido por la Dra. Alicia B. Gutiérrez y co-dirigido por el Dr. Héctor O. Mansilla, del que somos miembros. Para más información sobre la metodología de construcción del Espacio Social consultar (Gutiérrez y Mansilla, 2015), ya que sólo haremos una presentación breve aquí por no ser el objetivo de esta publicación.

${ }^{11}$ En función del modo en que se concibieron las estrategias del hogar, se seleccionó a un RH que reúne un conjunto de criterios establecidos por el equipo de investigación. En la mayoría de los casos el RH coincidió con el jefe de hogar autodesignado por el miembro del hogar entrevistado; pero en una proporción que varía entre el 20\% y el 25\% de los hogares se cambió el autodesignado jefe de hogar por un RH que responde al marco teórico de la investigación (Gutiérrez y Mansilla, 2015). 
fracciones construidas está fundamentada en la publicación de Gutiérrez y Mansilla (2015) y no nos adentraremos en ella para este artículo.

El programa SPAD (V5.5), por sus siglas en francés Systeme Portable pour l'Analyse de Données, permite la representación plana de una nube de puntos multidimensional. Esto es posible gracias al trazado de factores perpendiculares entre sí que capturan las mayores deformaciones de la denominada nube de datos. En este caso, se han representado los factores uno y dos (gráficos 4 y 5) que acumulan el mayor porcentaje de inercia.

Gráfico 4: El espacio social Gran Córdoba 2003.

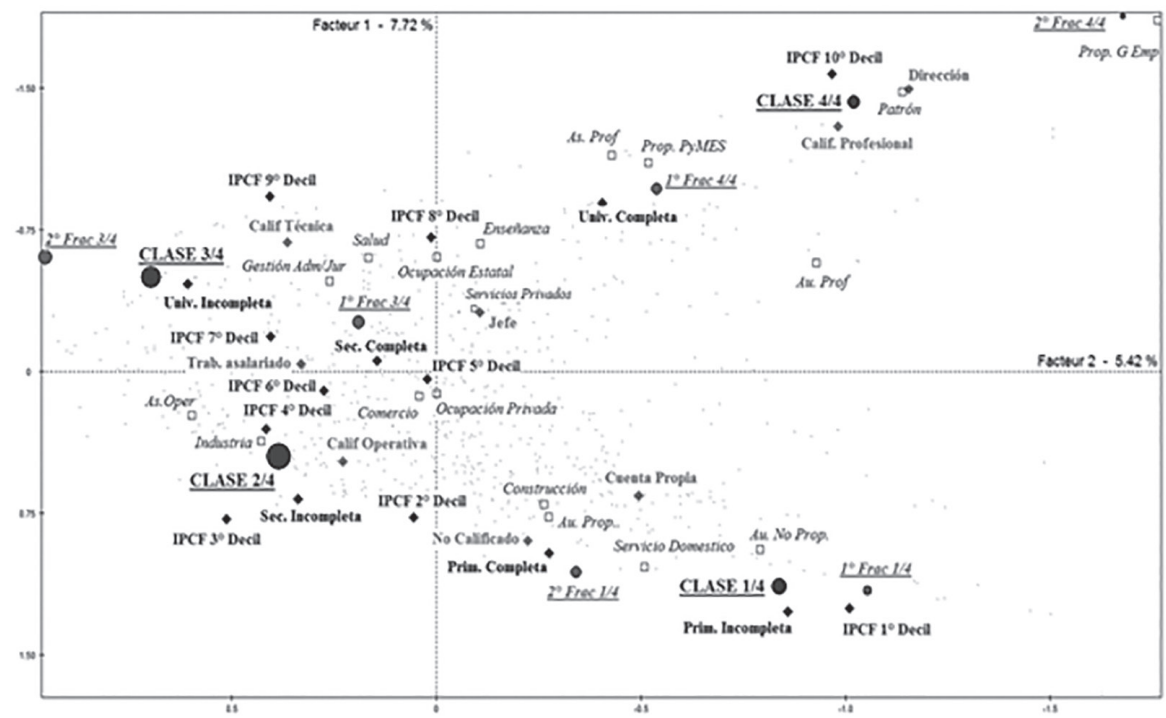

Fuente: Gutiérrez y Mansilla, 2015, p. 434.

En el espacio social se identificaron cuatro grandes clases: baja, media dominada, media dominante y alta. Para cada una de estas clases se construyeron dos fracciones de clase, a partir de un corte de 7 y 11 fracciones (Gutiérrez y Mansilla, 2015). Las familias de clases baja y media dominada en ambos años se caracterizan por tener una asociación significativa con niveles de estudio hasta secundario incompleto, trabajos no calificados o de calificación operativa y sus RH estar empleados o ser autónomos no profesionales en el servicio doméstico, la construcción, la industria o el comercio, principalmente. Son las que detentan los menores ingresos en las variables de ingreso consideradas para el estudio. 
Gráfico 5: El espacio social Gran Córdoba 2011

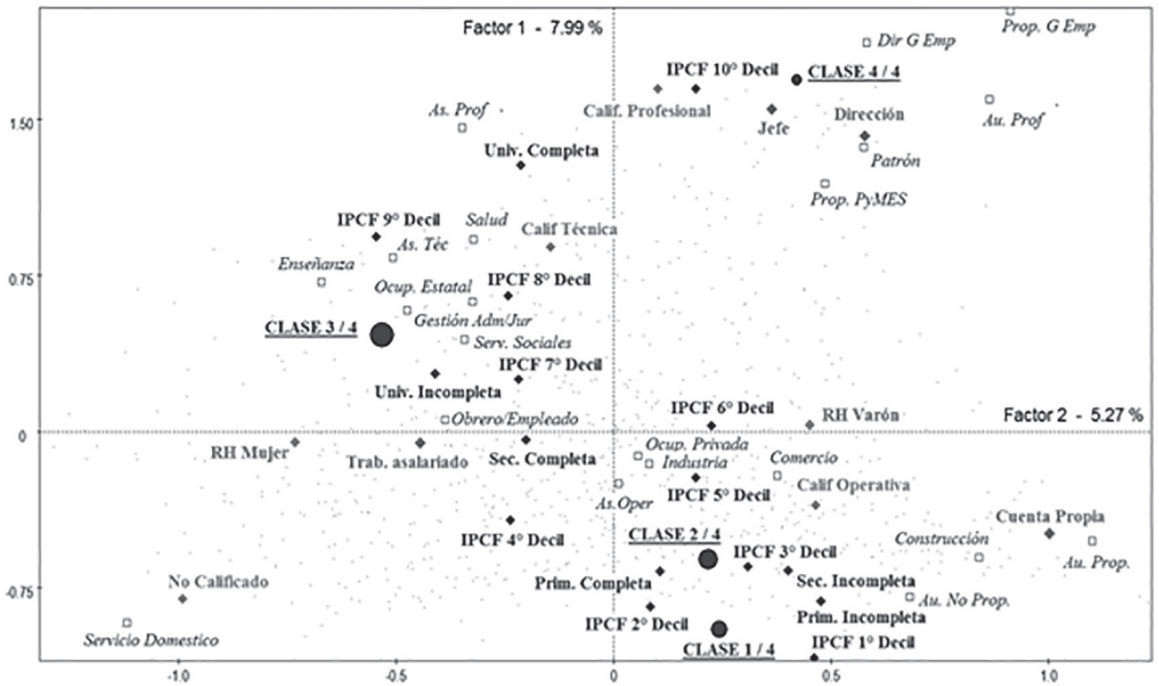

Fuente: Gutiérrez y Mansilla, 2015, p. 420.

Por otra parte, las familias que ocupan los cuadrantes superiores del espacio social, que pertenecen a las clases media dominante y alta, están significativamente asociadas a niveles educativos secundario completo, nivel superior universitario y no universitario en elevada proporción, llegando al nivel de posgrado. Estas familias están asociadas con los ingresos más altos del espacio social.

Dentro de la clase alta se identifican dos grupos, los profesionales con cargos directivos y los propietarios de Pymes y grandes empresas, en ambos casos varones con estudios universitarios completos y calificación laboral profesional. En la clase media dominante la primera fracción se asocia a mujeres separadas o divorciadas, asalariadas que trabajan en el estado en cargos de jerarquía media, con estudios universitarios completos. La segunda fracción se caracteriza por jóvenes solteros, mujeres y varones, asalariados con nivel universitario incompleto. Ambas fracciones tienen calificación laboral técnica. La clase media dominada, altamente homogénea, no distingue entre fracciones. En la clase baja la inserción laboral mediada por el género permite la diferenciación en dos fracciones, trabajadoras de servicio doméstico y varones trabajadores de la construcción.

La construcción de clases sociales para 2003 y 2011 permite dos niveles de análisis simultáneos. Por una parte, la descripción por clase de las con- 
diciones objetivas de acceso y finalización de los miembros de las diferentes familias de Córdoba y en segundo lugar observar los cambios que ocurren en el acceso y la finalización en 2003 y 2011.

Si tomamos los individuos de 25 años y más ${ }^{12}$, se aprecia la reducida proporción de miembros que finalizan el secundario para las familias de clase baja y media dominada y por oposición la elevada proporción de los miembros de las clases media dominante y alta que finalizan el nivel (ver tabla 3). La notable diferencia entre estos valores pone de relieve la gran dificultad que tienen las familias de clase baja y media dominada para acceder a los estudios superiores, y en particular a los universitarios.

Tabla 3: Finalización del secundario en individuos de 25 años y más por clase social construida, Gran Córdoba, 2003 y 2011.

\begin{tabular}{rccccccccc}
\hline & \multicolumn{2}{c}{ Clase Baja } & \multicolumn{2}{c}{$\begin{array}{c}\text { Clase Media } \\
\text { Dominada }\end{array}$} & \multicolumn{2}{c}{$\begin{array}{c}\text { Clase Media } \\
\text { Dominante }\end{array}$} & \multicolumn{2}{c}{ Clase Alta } \\
\cline { 2 - 9 } & 2003 & 2011 & 2003 & 2011 & 2003 & 2011 & 2003 & 2011 \\
\hline Hasta Sec. Comp. & $76.5 \%$ & $75.1 \%$ & $71.3 \%$ & $60.5 \%$ & $26.8 \%$ & $17.3 \%$ & $11.4 \%$ & $3.7 \%$ \\
\hline Sec. Comp. y más & $23.5 \%$ & $24.9 \%$ & $28.7 \%$ & $39.5 \%$ & $73.2 \%$ & $82.7 \%$ & $88.6 \%$ & $96.3 \%$ \\
\hline Total & $100.0 \%$ & $1000 \%$ & $100.0 \%$ & $100.00 \%$ & $100.0 \%$ & $100.0 \%$ & $100.0 \%$ & $100.0 \%$ \\
\hline
\end{tabular}

Fuente: elaboración propia en base a datos de la EPH 2003 y 2011.

Por otra parte, todas las clases aumentaron la terminalidad del secundario de 2003 a 2011, aunque de manera diferenciada. Se observa un mayor incremento de la finalización del secundario en las clases medias (dominada y dominante) y alta, con una mejoría que ronda el 10\% de los casos. En cambio, en la clase baja el crecimiento en la finalización es menor a un $2 \%$. En el contexto de democratización de la enseñanza secundaria, la obligatoriedad del nivel y las políticas públicas asociadas, la clase baja sigue sin poder aprovechar estas oportunidades. Son las clases medias y alta las que incrementan su presencia en el nivel. Y, lamentablemente, sigue pendiente la deuda con la clase baja.

Lo dicho hasta aquí explica la escasa participación de las clases baja y media dominada en el nivel universitario, que se puede apreciar también en el gráfico a continuación.

${ }^{12}$ El agrupamiento de 25 años y más responde al límite inferior del lustro más próximo a la edad teórica de egreso de una carrera de grado. 
Gráfico 6: Acceso y egreso universitario en individuos de 25 años y más, por clase social construida, Gran Córdoba, 2003 y 2011.

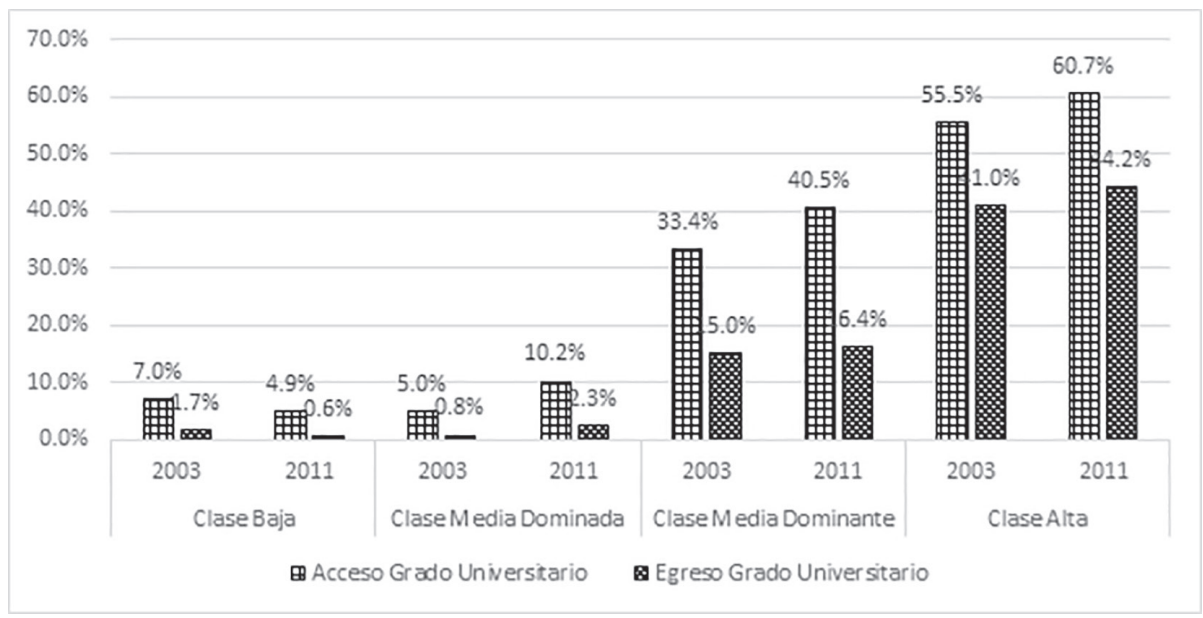

Fuente: elaboración propia en base a datos de la EPH 2003 y 2011.

A pesar de la leve mejora de las cifras de finalización del secundario de las familias de clase baja, el acceso a la universidad, ya escaso en 2003, disminuye en 2011, reduplicando la deuda social con estas familias. Su terminalidad es mínima, con valores por debajo del $2 \%$ de los casos. La situación es diferente para las familias de clase media dominada, en las que se observa un aumento en el acceso a la universidad y también en el egreso. Aun duplicando su acceso, la situación de estas familias sigue exponiendo la desigualdad educativa.

La clase media dominante también mejora su acceso a la universidad. Los mayores de 25 años que pertenecen a esta clase aumentan su acceso en 7 puntos, llegando a un 40,5\% del total. En cuanto al egreso, crece sólo 1,5 puntos, con lo que no llegan a egresar la mitad de los que accedieron oportunamente, como sí lo hacían en 2003.

En las familias de clase alta el fenómeno es diferente: el acceso también crece, en 5 puntos, y el egreso lo hace en 3 puntos. Las familias de clase alta son las que tienen una mejor proporción entre los miembros con acceso y los que egresan del nivel; más de dos de cada tres estudiantes de esta clase, egresan.

A continuación abordamos las diferentes estrategias de las familias frente al retraso en el cursado o a las dificultades que puedan presentarse, relacionándolas con el conjunto de determinaciones expuestas hasta aquí. 


\section{Prácticas y representaciones ante la dilación de los estudios}

Ante situaciones de dilación de los estudios, las estrategias que establecen las familias de Gran Córdoba son diferentes en función de su volumen de capital, su estructura y el estado de los instrumentos de reproducción disponibles. Estas estrategias, entendidas como prácticas y representaciones, implican para los estudiantes y sus familias un amplio espectro de posibilidades que va desde el abandono temprano hasta la permanencia a cualquier costo.

Para dar cuenta de estas prácticas y representaciones diferenciales, tomamos las entrevistas realizadas en el programa de investigación del que formamos parte (43 entrevistas a familias de Gran Córdoba), las realizadas en el marco de la investigación doctoral de Manuel Giovine (30 entrevistas a familias de clase alta de Gran Córdoba) y las sistematizadas para la investigación de maestría de Ana Antolin (286 entrevistas a alumnos que solicitan Continuidad de Estudios en la carrera de Medicina de la UNC).

De acuerdo al espacio social construido, las familias se estructuran en dos grandes grupos: uno conformado por las familias de clase media dominante y alta, con elevada proporción de acceso a la universidad y otro formado por las familias de clase media dominada y baja que, por oposición, tienen una proporción muy reducida de miembros con acceso al nivel universitario, como mostramos en el apartado anterior.

Es decir que el acceso diferenciado al nivel universitario está relacionado significativamente con la clase social construida. Las entrevistas realizadas nos permiten identificar las prácticas y representaciones diferenciadas por clase y fracción de clase frente a la dilación en los estudios universitarios. Cuando se da esta situación de dilación o probable abandono, algunas familias o individuos articulan múltiples estrategias de permanencia en el nivel.

La investigación cualitativa nos permitió identificar algunas de ellas, que hemos agrupado como estrategias de persistencia y estrategias de sustitución. Las estrategias de sustitución predominan dentro de las clases media dominante y alta, e implican el cambio de carrera dentro de la misma universidad, el cambio de universidad o cambio de nivel buscando tecnicaturas universitarias $\mathrm{u}$ otras carreras de nivel superior. Por su parte las estrategias de persistencia que predominan en las clases media y baja implican mantenerse en la carrera aunque con avances mínimos, tales como la sola rematriculación o aprobar pocas materias por año, entre otros. 
En las familias de clase media dominante y alta, el acceso a la universidad es algo esperado y promovido para todos sus hijos. Las condiciones de acceso a la universidad comienzan a ser construidas en el núcleo familiar desde el primer momento, orientando el proceso de escolarización previo. Esto logra que el tránsito a los estudios universitarios sea percibido con "naturalidad". En contrapartida, el retraso en el cursado y el abandono son vividos con frustración y rechazo y las estrategias frente a la dilación en la carrera son articuladas por las familias cuanto antes.

Por su parte, en las familias de clase media dominada y baja, donde el acceso a la universidad es menor y las probabilidades de egreso son muy bajas, el ingreso a cualquier carrera universitaria es significado como un progreso, que implica el reconocimiento familiar. Al mismo tiempo, las propias condiciones materiales de existencia de las familias se presentan como una limitación al acompañamiento que puedan brindar.

En las familias de clase alta las estrategias de permanencia surgen predominantemente ante el fracaso académico. En cambio, en las familias de las restantes clases, las estrategias de permanencia pueden aparecer ante diversos factores, como dificultades económicas, familiares y académicas. En particular en las familias de clases media dominada y baja, dadas sus condiciones objetivas, el sólo acceso a la universidad ya implica articular múltiples estrategias para lograr la permanencia de sus miembros.

Los costos que supone una carrera en una universidad estatal para una familia de clase dominante, media o alta, cualquiera sea la carrera elegida, pueden ser afrontados sin mayores dificultades económicas. Sin embargo, cuando sus hijos asisten a universidades privadas, los costos de los estudios pueden llegar a tener una mayor repercusión sobre la economía familiar. En las familias de las restantes clases construidas la cuestión económica es cuidadosamente controlada. Cualquier desajuste económico-financiero a nivel macrosocial o familiar puede llevar al estudiante a ingresar tempranamente al mercado laboral, con posibilidades de retraso en el cursado o, en el peor de los casos, abandono de la carrera.

\section{Clase alta dominante: $\mathrm{RH}$ empresarios y profesionales directivos}

Las familias de clase alta son las que detentan un mayor volumen de capital global. En general para todas estas familias los estudios universitarios se configuran no tanto como un horizonte deseable, sino como un objetivo 
concreto y esperable a no muy largo plazo para sus hijos. Las familias están dispuestas a invertir grandes volúmenes de capital, no sólo económico, de manera sostenida en el tiempo.

Para precisar un poco más sobre la dilación de los estudios y el posible abandono (infrecuente para las familias de esta clase, en nuestras muestras) es necesario distinguir por fracción de clase alta construida, entre los empresarios y los profesionales directivos (Giovine, 2015).

En las familias de los empresarios, la finalización del secundario y el acceso a la universidad son vivenciados como una transición esperada. Las opciones en esta fracción están mediadas por cuestiones vocacionales, por el tipo de universidad o por las perspectivas de inserción en la empresa. El título universitario es buscado no sólo como un instrumento para mejorar el nivel de ingresos sino también para legitimar una posición en la empresa, que en algunas casos indica una posición en la familia.

En la fracción de los profesionales directivos, donde el capital cultural es el elemento estructurante de su posición dominante en el espacio social, el paso por la universidad tiene una impronta marcada por el deber y el fracaso es percibido como un riesgo de desclasamiento. Implica también elecciones conservadoras de carrera, volcándose hacia aquellas tradicionales como derecho, medicina, ingeniería y en menor medida ciencias económicas, cuyos títulos habilitan a los espacios laborales que de un modo u otro ya pertenecen a la clase.

En la significación de las familias de clase alta, la UnC es considerada como una opción para la educación de sus hijos porque goza de ciertos prestigio y distinción. Su antigüedad, enraizada en la historia misma de Córdoba, favoreció la formación de la clase alta de la provincia y del interior del país. Y aparece en la formación de los padres, abuelos y bisabuelos de los entrevistados.

No obstante, los recientes procesos de masificación y democratización (Brunner, 2012; Rama, 2009), sumados a la diversificación de las ofertas estatal y privada, han tenido como consecuencia que los aspirantes a ingresar a la UNC de las familias de clase alta de primera fracción, encuentren mayores dificultades al momento del acceso y durante el proceso, que los llevan a considerar como opción a las universidades privadas.

Los alumnos que logran sortear la dificultad en el acceso suelen enfrentarse a dificultades durante los primeros años de carrera, que no son propiamente problemas de aprendizaje y que podemos asociar con los espacios de contención propios de las escuelas privadas donde se los identificaba personalmente a ellos y a sus familiares.

Es el caso de Julieta, que egresó de una escuela privada laica bilingüe, donde estudió por dieciséis años y donde estudian actualmente sus dos her- 
manos. Al describir este fenómeno de contraste, expresa "me pasó por encima un tractor", refiriéndose a cierta incomodidad y aturdimiento en el espacio físico, lleno de carteles de agrupaciones políticas, falta de individuación que hacía que sus profesores no la reconozcan por su nombre y la masificación, que le impedía establecer relaciones duraderas con una misma persona.

En los casos de jóvenes de clase alta que logran superar estas barreras, continúan sus estudios hasta finalizarlos. Hacerlo por medio de valores que ellos recuperan en términos de perseverancia y esfuerzo, es resignificado como "mérito". Su trayecto en la universidad estatal, finalicen o no sus estudios, aparece en las entrevistas como que les "da calle", refiriéndose a la capacidad de relacionarse con individuos de otras clases sociales y desenvolverse en una institución pública.

En los casos en que los entrevistados abandonan la universidad estatal, optan por continuar sus estudios en universidades privadas como la Universidad Católica de Córdoba y la Universidad Blas Pascal u otras de Buenos Aires y del extranjero. Para las carreras profesionales tradicionales, cambiarse desde la universidad estatal a la privada implica, en el discurso de los entrevistados, condiciones de cursado más adecuadas a sus necesidades de tiempo, de exigencias, un acceso más directo a los docentes y un trato más personalizado. Reconocen sin embargo el "sacrificio" que significa dejar la universidad estatal, donde se han formado sus padres.

Para las familias de los referentes empresarios la universidad estatal tiene poco para ofrecer e implica mucho costo, sobre todo en probabilidades de fracaso y abandono. La opción por universidades privadas se realiza tempranamente y la oferta estatal rara vez es parte de sus opciones.

\section{Clase media dominante: $\mathrm{RH}$ universitarias en el estado y universitarios incompletos asalariados}

En las familias de clase media dominante el acceso a la universidad de sus miembros representa una oportunidad de promoción social y educativa. Aunque por debajo del acceso y la graduación de las familias de clase alta, entre 2003 y 2011 las familias de clase media dominante incrementaron el acceso y la graduación al nivel universitario. Por eso en su significación el acceso a la universidad es visto como posible y esperable. Las familias en las que alguno de sus miembros asiste a la universidad, cuentan en su red con familiares universitarios, por lo general uno de los padres o familiares próximos, instalando la disposición a estudiar una carrera universitaria o de nivel superior. 
Entre las familias de esta clase se identifican dos fracciones. La primera fracción incluye preponderantemente mujeres con puestos técnicos en el estado, en educación y salud, con nivel universitario completo. La segunda fracción, con un mayor volumen de ingresos, incluye mujeres y varones jóvenes solteros, con calificación técnica y universitario incompleto. En ambos casos la composición de los hogares, madres separadas o divorciadas y jóvenes solteros, incide en las prácticas diferenciales de cada fracción.

Probablemente la principal diferencia entre la primera y la segunda fracciones de la clase media dominante sea que las familias de la primera fracción, con menores recursos, buscan continuar dentro de la universidad estatal, con carreras alternativas o cambiar entre universidades estatales, permaneciendo en el nivel. En cambio, la segunda fracción, por su mayor capital económico puede acceder a la universidad privada. Estas familias buscan con mayor frecuencia y cierta celeridad alternativas como las de continuar sus estudios en universidades privadas, como la UBP y la SxXI, universidades que cuentan con modalidades específicas para trabajadores.

Los hijos de estas familias consideran como primera alternativa el acceso a la UNC. Esta práctica adquiere sentido en el contexto de una generación de padres egresada, en su mayoría, de allí, y la movilidad social propiciada por las carreras liberales en la clase media cordobesa.

En los casos en los que se produce una dilación en los estudios, ésta aparece principalmente ante dificultades para alcanzar las exigencias académicas de las carreras universitarias. En las entrevistas se mencionan diversos factores asociados a la dilación, como ser cuestiones de salud, pérdida de alguno de los miembros del núcleo familiar, la temprana inserción en el mercado de trabajo, la presencia de familiares a cargo o la llegada de hijos al núcleo familiar, o momentos mismos del ciclo macroeconómico que comprometen la condición laboral del sostén del hogar. Estos factores inciden en mayor o menor medida en la permanencia y en las estrategias que se puedan generar.

En muchos casos, el primer año en la universidad es determinante para el abandono (Ambroggio, 2012) o la prosecución de los estudios. El retraso pone en crisis la continuidad misma en la carrera, por aumentar la probabilidad de abandono o de dilación. Sin embargo, la promesa de egreso de una carrera universitaria, en algunos casos acompañada de la inserción temprana en el mercado de trabajo en puestos afines a la carrera elegida, funcionan como alicientes para la continuidad en los estudios.

En los casos en que la dilación persiste, a través de lo que llamamos estrategias de persistencia los estudiantes redefinen sus metas y ajustan a sus avances a sus posibilidades. Así sostienen la esperanza de avanzar en la ca- 
rrera y superar los factores que los condicionan, los que en sus discursos muchas veces aparecen invisibilizados y adjudicados a las "materias filtro".

Cuando los estudiantes optan por cambiar de carrera, lo que forma parte de las estrategias de sustitución, lo hacen por otras que impliquen menos años de estudio, donde se reconozcan algunas materias del trayecto académico recorrido, preferentemente dentro de la misma universidad.

Otro grupo continuará sus estudios pero en institutos de nivel superior. Aquí expresan la necesidad de finalizar su formación y las dificultades que implicaba cursar en la universidad, asociadas principalmente a la organización académica. No obstante, todos reconocen la salida del nivel universitario con pesar, como una pérdida y en algunos casos como una deuda con la familia.

Entre quienes permanecen en el nivel universitario identificamos diferentes recorridos involucrando la opción por otras universidades. Aquellos que cursaban carreras de ingeniería en la UNC, continúan en la Universidad Tecnológica Nacional carreras de ingeniería o tecnicaturas. Quienes pasan de la UNC a universidades privadas, optan principalmente por carreras dentro de la Universidad sxxi o a la Universidad Blas Pascal, donde se encuentran además con otros equipamiento e infraestructura, diferentes modalidades de cursado, otros horarios de cursado, mayor proximidad con los docentes y principalmente otra contención.

\section{Clase media dominada: RH operarios asalariados adultos}

En esta clase la proporción de hogares con estudios universitarios, completos e incompletos, es notablemente menor a las clases ya presentadas. Aún así, entre 2003 y 2011 se registra una mejora en el acceso y un leve incremento en el egreso de los estudios universitarios.

Para las familias entrevistadas de clase media dominada, el acceso a la universidad representa una mejora en la calidad del empleo, la jerarquía laboral y los ingresos. Formar parte de la universidad es vivenciado como una oportunidad, no sólo para el acceso a nuevos conocimientos, sino también para el establecimiento de otras redes sociales y la participación en nuevas prácticas culturales.

Para la elección de la carrera aportan su carga simbólica y "motivacional" los recorridos universitarios de familiares $u$ otros significativos, aunque sean incompletos. Entre los alumnos provenientes de hogares con padres mecánicos, metalúrgicos y constructores, empleados de banco, de comercio, 
administrativos, las elecciones de carrera no se separarán ampliamente de las ramas de actividad económica de sus parientes, sino que buscarán sumar a la trayectoria laboral familiar la calificación profesional que otorga el título universitario. Se busca una mejora en las condiciones ocupacionales: trabajo registrado, seguridad social y por supuesto ingresos, que se traducen en mejoras en las condiciones de vida como acceso a tecnologías, viajes, casa propia y vehículo.

Las familias logran la permanencia de sus miembros en la universidad cuando confluyen algunas condiciones propias y de la sociedad. La finalización de los estudios secundarios, cierta estabilidad económica, ideas y representaciones en el entorno familiar o laboral cercano sobre los estudios de nivel superior, entre otras condiciones, aparecen como fundamento material y simbólico en los hogares que logran enviar y mantener a sus hijos en la universidad.

La estabilidad económica, social y familiar en la clase media dominada aparece en las entrevistas como condición de posibilidad para financiar los costos de una carrera universitaria. Especialmente en aquellas que demandan invertir en instrumentos y materiales de trabajo, o disponer de horarios amplios para cursar, ya que en estas condiciones difícilmente podrán aportar al presupuesto familiar, al tiempo que demandan recursos específicos para sostener el cursado. En este contexto, el carácter gratuito y abierto de las universidades estatales cordobesas es crucial a la hora de seleccionar la institución donde estudiar.

En las familias de esta clase el acceso al nivel universitario no está garantizado para todos sus miembros, sólo aquellos hijos que tuvieron en el nivel secundario un mejor desempeño académico y mientras sus familias transiten una coyuntura económica favorable, pueden "tener la suerte de estudiar".

En aquellas familias con más de un hijo en la universidad, ante la sobrecarga económica, suelen generarse tensiones que pueden dar por resultado que uno de ellos deba pasar, gradualmente, al mercado de trabajo, por lo general alejándolo del estudio. En estas circunstancias se observan casos donde los estudiantes deambulan de changa en changa, para no comprometer los horarios de cursado u optan por trabajar de noche para poder cursar de día.

En la medida en que se dilatan los estudios, el paso de los años pone al estudiante frente a nuevas responsabilidades, como compartir el cuidado de miembros de su familia, o su propio proyecto de paternidad/maternidad. Asumir a otros familiares a cargo afecta su ritmo de cursado, las horas de estudio y consecuentemente la presentación a exámenes. Cuando el cuidado de familiares requiere dedicación plena el abandono de la rutina de estudio, provisorio en un primer momento, se transforma en definitivo. 
En las parejas de estudiantes, en ocasiones sucede que mientras uno de sus miembros posterga sus estudios, el otro avanza en la carrera. Esto genera ciertos conflictos, pero también posibilita que, una vez finalizada la carrera, el miembro que postergó sus estudios se reincorpore, aunque casi nunca en las mismas condiciones.

Los relatos permiten elaborar una aproximación a las estrategias que se establecen para permanecer en la universidad, advirtiendo de qué manera el deseo de progresar y la fragilidad de las condiciones se conjugan para que quienes asuman estos estudios, persistan en ellos. Todo fracaso es sufrido; toda demora, resistida y siempre asumida como provisoria. Cualquier mejora en las condiciones económicas o laborales es argumento para retomar la carrera.

En esta clase no hay muchas opciones a la hora de reformular su estrategia o de optar por universidades privadas, por eso se persiste en la que ya se inició; a esto llamamos estrategias de persistencia. Se advierte aquí cómo difiere de la lógica de las clases dominantes, en las que predominan las estrategias de cambio.

\section{Clase baja: RH empleadas domésticas y obreros de la construcción}

Como ya se anticipó, la participación de esta clase social en la población con estudios universitarios, completos e incompletos, es mínima, no llegando al $2 \%$ del total.

A pesar de la disponibilidad de numerosas instituciones universitarias, y el carácter gratuito de la mayoría de las carreras que se ofrecen, el acceso al nivel universitario para las familias de esta clase sigue siendo muy limitado, en principio porque la mayoría no logra terminar el secundario.

Entre los agentes entrevistados, la universidad difícilmente aparece en el horizonte de posibilidades y deseos, porque alcanzar el título secundario ya es todo un logro. Pero aun si ese título se obtiene, las opciones se orientan hacia carreras cortas (gestión, recursos humanos, enfermería), capacitaciones laborales estructuradas (estética, $\mathrm{CNC}$, instalaciones industriales o domiciliarias) de pronta salida laboral o que pueden cursarse al mismo tiempo que se trabaja, más que hacia carreras profesionalizantes de largo aliento. En estos casos, el universo de significados que da sentido a la elección de estudios universitarios toma forma a partir de dos situaciones: o la inserción laboral de los padres o su propio contexto laboral. 
Las urgencias económicas, de subsistencia, se interponen ante la posibilidad de encarar estudios superiores. Así, aunque un miembro de estas familias logre terminar sus estudios secundarios e ingrese a la universidad, debe salir al mercado de trabajo para poder mínimamente sostenerse, cuando no contribuir a la economía familiar. En el mejor de los casos la familia articula múltiples estrategias de permanencia para sostener sus estudios, al menos hasta obtener un título intermedio o de tecnicatura.

Sin embargo, en la mayoría de los casos no logran finalizar sus estudios. Los avances son mínimos y la estrategia predominante consiste en rematricularse año a año, sosteniendo la condición de estudiante universitario. Estas estrategias de persistencia se pueden interpretar como formas de resistencia frente a un conjunto de condicionantes que termina siendo expulsivo de las instituciones.

En el discurso de los agentes, el hecho de rematricularse sin avanzar en el cursado no es percibido como un tiempo muerto; no se pierde contacto con la carrera, se busca estar actualizado y no desvincularse de los espacios de práctica que hacen a la profesión. En definitiva, se habita el tiempo de espera por mejoras en las condiciones económicas o institucionales para encarar, nuevamente, la carrera hasta finalizarla.

\section{Conclusiones}

En este artículo se ha mostrado la evolución de la matrícula universitaria desde fines del siglo xx, y su crecimiento da cuenta del proceso de democratización del acceso a la educación universitaria en Argentina y Córdoba, un ideal que enarboló la Reforma Universitaria de 1918 y es retomado desde la vuelta a la democracia en 1983. Sin embargo, el egreso no ha crecido significativamente desde entonces y ha aumentado la permanencia de los estudiantes en las universidades, lo que denominamos dilación en los estudios. Y además, no obstante la apertura de las instituciones universitarias, ni el acceso, ni el egreso, ni la permanencia en los estudios llegan equitativamente a todas las familias del espacio social, siendo las clases dominadas las menos favorecidas.

Ante la dilación en sus estudios universitarios y en la búsqueda del título universitario, los estudiantes y sus familias de Gran Córdoba articulan estrategias diferenciadas según la posición en el espacio social y la trayectoria familiar, que llamamos estrategias de permanencia. Al interior de las estrategias de permanencia hemos podido identificar dos grandes grupos 
que denominamos estrategias de sustitución y de persistencia. Las primeras aparecen asociadas a las familias de clase dominante y las segundas a las familias de clase dominada, dando cuenta de una relación con cierto volumen y estructura de recursos, una trayectoria y el estado de los instrumentos de reproducción que operan como límites y posibilidades de las estrategias.

Las estrategias de sustitución consisten en el cambio de carrera y/o institución educativa en pos de asegurar el egreso y se articulan con cierta premura frente a las situaciones de dilación de los estudios. La sustitución se puede dar entre instituciones estatales o privadas, ya sean de nivel superior o universitario. Se establecen predominantemente en familias de clase alta y clase media dominante, que cuentan con los recursos económicos como para afrontar estos desplazamientos. En las familias de clase alta, ocurre con mayor frecuencia la opción por las universidades privadas; en cambio, en las de clase media dominante se opta principalmente por instituciones estatales o en menor medida universidades privadas con modalidades de cursado más accesibles.

Por su parte, las estrategias de persistencia consisten en sostener el cursado por todos los medios, se ralentiza el avance en la carrera esperando una mejora en las condiciones que dificultan su progreso, o que el tiempo ayude a consolidar los conocimientos, o que ocurra un cambio extraordinario que permita reanudar el ritmo y egresar. Esta espera, con el paso del tiempo, suele tener efectos no deseados que terminan condicionando aún más la situación de los estudiantes. Las estrategias de persistencia son establecidas principalmente por las familias de clases baja y media dominada, cuyo margen para cambiar de carrera o universidad es mucho menor.

El acceso a la universidad es visto como algo "natural" y "esperable" por las familias de clases alta y media dominante, y la dilación de los estudios es tomada como un fracaso, una vergüenza y una deuda. En las familias de clases media dominada y baja, en cambio, el acceso es visto como una oportunidad de promoción social y económica, de crecimiento personal y de acceso a un nuevo mundo cultual que, dada su condición de acceso siempre precaria, de algún modo siempre prevé cierta contingencia y la dilación de los estudios universitarios. No obstante ello, la experiencia de la dilación es para todos los estudiantes y sus familias una experiencia negativa y dolorosa; en lo que difieren ampliamente las familias, según su posición en el espacio social, es en las estrategias que pueden articular ante esta situación. 


\section{Referencias}

Acosta, F. (2009). Re-configuración de la escuela secundaria frente al ingreso masivo de jóvenes de sectores populares. Revista de Pedagogía, 30(87), 217-246.

Ambroggio, G. A. (2012). El primer año en la universidad y la permanencia en la carrera. Cuadernos de Educación, 1(1), 133-143.

Bourdieu, P. (1990). Espacio social y génesis de las clases. Sociología y cultura, $\mathrm{s} / \mathrm{d}(\mathrm{s} / \mathrm{d}), 281-309$.

Brunner, J. J. (2012). La idea de universidad en tiempos de masificación. Revista iberoamericana de educación superior, 3(7), 130-143.

Brunner, J. J., y Miranda, D. A. (2016). Educación superior en Iberoamerica: informe 2016. Santiago de Chile: CINDA.

Chiroleu, A. (2009). La democratización del acceso a la universidad: de la ampliación de oportunidades a la inclusión. A, 90, 99-116.

Dirección Nacional de Información y Estadística Educativa, Argentina. (2016). La cobertura del nivel inicial en Argentina. Situación y tendencias en contexto. DINIEE.

Ennis, H. M., y Porto, A. (2001). Igualdad de Oportunidades e Ingreso a la Universidad Pública en la Argentina. Documento de Trabajo No 30. FCE-UNLP.

García de Fanelli, A. M. (2005). Acceso, abandono y graduación en la educación superior argentina (Educación superior. Acceso, permanencia y perfil social de los graduados comparados con los egresados de la educación media. Debate No. 5). Buenos Aires: SITEAL.

García de Fanelli, A. M. (2015). Inclusión social en la Educación Superior Argentina: indicadores y políticas en torno al acceso ya la graduación. Social Inclusion in Argentina Higher Education: Indicators and Policies Regarding Access and Graduation. Páginas de Educación, 7(2), 124-151.

Giovine, M. (2015). Condicionamientos educativos de los sectores dominantes en Gran Córdoba: cambios y transformaciones 2003-2011. Revista de la educación superior, XLV(177), 119-145.

Gorostiaga, J. M. (2012). Las políticas para el nivel secundario en Argentina: ¿hacia una educación más igualitaria? Revista Uruguaya de Ciencia Política, 21(1), 141-159.

Gutiérrez, A. B., y Giovine, M. (2017). Access to Higher Education ante the Reproduction of Inequalities in Córdoba, Argentina. Rassegna Italiana di Sociologia, 58(2), 381-417.

Gutiérrez, A. B., y Mansilla, H. (2015). Clases y reproducción social: el espacio social cordobés en la primera década del siglo XXI. Política y Sociedad, 52(2), 409-441.

Instituto Nacional de Estadísticas y Censos. (2018) Anuario Estadístico de la República Argentina 2016. Buenos Aires: INDEC.

Jacinto, C. (2006). Los protagonistas de la expansión de la educación secundaria. Anales de la educación común. Educación y trabajo, 2(5). 
Jiménez Zunino, C. I., y Giovine, M. (2017). Terminar el secundario en Córdoba: desigualdad educativa y nivel medio en la última década. Páginas de Educación, 10(2), 21-43.

Ministerio de Educación de la Nación. (2011). Anuario de Estadísticas Universitarias - Argentina 2011 (p. 378). Buenos Aires: Secretaría de Políticas Universitarias.

Miranda, E. (2013). De la selección a la universalización: Los desafíos de la obligatoriedad de la educación secundaria. Espacios en blanco. Serie indagaciones. 23, 9-32.

Nobile, M. (2016). La escuela secundaria obligatoria en Argentina: Desafíos pendientes para la integración de todos los jóvenes. Última década, 44, 109-131.

Palermo, A. I. (2006). El acceso de las mujeres a la educación universitaria. Revista Argentina de Sociología, 4(7), 11-46.

Rama, C. (2009). La tendencia a la masificación de la cobertura de la educación superior en América Latina. Revista Iberoamericana de Educación. 50, 173-195.

Rivas, A., Vera, A., y Bezem, P. (2010). Radiografía de la educación argentina. Buenos Aires: Fundación CIPPEC, Fundación Arcor, Fundación Roberto Noble.

Stasiejko, H., Krauth, K. E., Pelayo Valente, J. L., Mirko, M. A., y Angellotti, C. E. (2013). Indagación de las concepciones acerca de la dilación en el estudio durante el ingreso a la Universidad. En $V$ Congreso Internacional de Investigación y Práctica Profesional en Psicología XX Jornadas de Investigación Noveno Encuentro de Investigadores en Psicología del MERCOSUR. Facultad de Psicología-Universidad de Buenos Aires. 1, 304-307.

Suasnábar, C. (2009). La Reforma Universitaria de Córdoba de 1918: una mirada histórica de la relación entre intelectuales, universidad y política en la Argentina. Práxis Educativa (Brasil), 4(1), 51-61. 